\title{
Una mirada sobre las actividades culturales del COAA en 2019
}

\author{
José Ramón Puerto Álvarez \\ Vicedecano del COAA
}

RESUMEN

En este artículo se pretende ofrecer una visión global de las actividades culturales que desarrolló el Colegio Oficial de Arquitectos de Asturias a lo largo de año 2019, de las cuales es necesario destacar tanto las exposiciones de Arquitectura como las de otras especialidades artísticas, las conferencias de arquitectos de ámbito regional y nacional o los viajes culturales. Como actos principales del periodo merecen reseñarse los actos de entrega de los premios Castelao a Nicolás Arganza García y COAA+10 por la propuesta de Casas Cuartel de la Guardia Civil de Pola de Lena (1982), Piedras Blancas (1982) y Luarca (1984) realizadas por el estudio de Jose Manuel Caicoya y Manuel García García.

\section{PALABRAS CLAVE:}

Arquitectura, COAA, Premio Castelao, Premio COAA + 10 .

\section{ABSTRACT:}

This article aims to provide a global view of the cultural activities that were carried out by the Official College of Architecture of Asturias (Colegio Oficial de Arquitectos de Asturias) throughout the year 2019, from which is necessary to highlight the expositions about architecture and other artistic specialties, the conferences of architects of regional and national scope or the cultural trips. As main acts of this period a well-deserved mention to the Castelao awards conferred to Nicolas Arganza Garcia y COAA+10 for the proposal of Civil Guard's Barrack Houses of Pola de Lena (1982), Piedras Blancas (1982) y Luarca (1984) made by Jose Manuel Caivoya and Manuel Garcia Garcia' studio. 
El actualizar conocimientos en cualquier profesión hoy en día es algo obligado y en una tan compleja como la Arquitectura no podía ser menos: nuevas técnicas, materiales o normativas aparecen de forma continua y es necesario realizar ese esfuerzo constante de puesta al día para seguir ofreciendo en nuestro trabajo la mayor profesionalidad.

Pero si sólo se dedica tiempo a actualizar conocimientos que tengan una respuesta inmediata en la profesión es fácil que una parte de nuestro yo creativo quede sin el sustento adecuado para seguir realizando proyectos que crucen la línea que diferencia construcción y Arquitectura. Por eso es necesario nutrir esa parte que tenemos latente los arquitectos y para ello es vital que no nos encerremos exclusivamente en nuestro ámbito profesional y podamos aprender, admirar y asimilar lo que otros compañeros u otras especialidades artísticas realizan y para ello a lo largo de 2019 hemos organizado desde el Colegio Oficial de Arquitectos de Asturias distintas actividades que buscan complementar eso que el día a día no nos aporta. Así, entre otros actos, se realizaron varias exposiciones que llenaron con nuevos aires el espacio de nuestras sedes colegiales de Oviedo y Gijón.

En marzo, uno de nuestros grandes artistas asturianos, el escultor Francisco Fresno, tuvo la generosidad de presentar en nuestra sede de Marqués de Gastañaga, 3, de Oviedo una selección de obras que hicieron un repaso a su trayectoria. Allí se pudieron ver maquetas y fotografías de esculturas que han pasado a ser referencia en las ciudades donde se colocaron (fundamentalmente Gijón, donde reside), pero también pinturas de distintas etapas, sus primeros pasos en la profesión y obras terminadas que fueron importantes en los momentos de su realización. Una conferencia del historiador de arte Juan Carlos Aparicio puso broche a esta exposición con la visión del crítico que mira al artista. Dos meses después otro escultor, Enrique Cifuentes, volvía a llenar los mismos espacios de una manera muy distinta: desde el vestíbulo de ingreso unas piezas construidas con alambrón adoptaban una forma similar a un "donut" y colonizaban escaleras y parte de las salas de exposición acompañando a otras obras donde la importancia de las texturas de los materiales utilizados (madera, pizarra, granito) tenían gran importancia en el aspecto final de las mismas. En el centro del espacio expositivo un tótem de granito de más de 2 metros de altura presidía majestuoso el lugar. Casi coincidiendo en el tiempo, pero en este caso en nuestra sede de Gijón, Alfonso Pire, filósofo de profesión y escultor de pasión, presentaba ST-275 STEEL, una exposición que adoptó el nombre industrial del material con el que está hecha y que según el autor "investiga sobre el vacío a partir de una reducción formal en la expresión respecto a trabajos anteriores".

No fueron solo escultores los que poblaron nuestras salas con sus trabajos y así, tras la inauguración en la Noche Blanca de Oviedo durante el mes de octubre el COAA tuvo la oportunidad de disfrutar de la exposición "Sobre mujeres artistas", muestra de la que fue comisario Santiago Martínez y en la que se mostró el trabajo de 22 creadoras asturianas que mostraron su trabajo en campos tan diversos como la pintura, la fotografía, la escultura, la obra gráfica o la tradición textil. Sirva citar a María Jesús Rodríguez, Mónica Dixon, María Álvarez, Esperanza D’Ors, María Braña, Soledad Córdoba o Jezabel Rodríguez entre ese elenco de creadoras que, lamentablemente, sólo pudieron estar expuestas hasta finales de octubre al tener otro compromiso adquirido con la Universidad de Oviedo en una actividad conjunta que pretendía celebrar el doble aniversario de la Bauhaus y la caída del muro de Berlín. Una exposición de caricaturistas griegos nos trajo la realidad de otros muros, estos no físicos, que se siguen estableciendo en una sociedad cada vez más dividida y donde un mar de vida y cultura como el Mediterráneo se acaba convirtiendo en un triste final para cientos de personas que intentan cruzarlo en unas condiciones precarias.

También hubo tiempo para exponer, como no, Arquitectura en dos ocasiones, pudiendo presentarse en Oviedo la muestra que Héctor Blanco había realizado anteriormente para la Fundación Alvargonzález en la que se mostraban todas las obras asturianas que se habían publicado en la revista Arquitectura del COAM. Se mostraron trabajos de Castelao, de Marín, de los Somolinos, de Vaquero y de otros muchos compañeros que tuvieron la posibilidad de construir en una época en que los tiempos tenían una cadencia más lenta que los actuales con resultados que nos siguen admirando. También se montó en la sede de Gijón la exposición de obras presentadas y premiadas a la XXIV edición de los premios Asturias de Arquitectura. La obra vencedora, la pantalla contra el viento del Musel, de Abel Alonso y Bibiana García, tuvo el reconocimiento 
que merecía, así como los premiados y accésit en las distintas categorias convocadas, como fueron los trabajos realizados en la Rehabilitación del Edificio Antigua Escuela de Comercio de Gijón de los arquitectos Javier Uría de la Fuente, María López Castro, Mónica Costales Rodríguez, Ramón Ruiz Fernández y Jorge Alonso Nicieza. También ocuparon un puesto de honor la vivienda unifamiliar aislada Cabaña $Z$ realizada en Tapia de Casariego por Jose Ángel Vior Alonso y la vivienda unifamiliar aislada en Pancar de los arquitectos Ester Roldán y Víctor Longo. El proyecto que se alzó con el Premio Asturias ganó también la categoría Otras Arquitecturas, siendo accésit en la misma la página web "Arquitectura de Asturias" que se ocupa de difundir las mejores obras de la reciente arquitectura de nuestra región y está compuesta por un grupo de jóvenes arquitectos.

No podía faltar en nuestra programación de actos culturales un ciclo de conferencias en las que destacados arquitectos del ámbito regional o nacional nos mostraran sus obras, siendo nuestro compañero Pedro Quero el que inició el año, mostrando algunos trabajos realizados en Asturias y otros en Edimburgo donde es profesor en la Facultad de Arquitectura. Tras él fue Javier Vellés, profesor de la ETSAM con Francisco Javier Sáenz de Oiza, quien habló sobre el desaparecido maestro de arquitectos aprovechando el 50 aniversario de la construcción del Colegio de la Milagrosa de 0viedo, única obra realizada en Asturias por el arquitecto tudelano. Nuestro Colegio profesional participó en varios actos que la comunidad educativa realizó con el alumnado para celebrar la efemérides, concluyendo en octubre los mismos con la colocación por nuestra parte de una placa de la Fundación DOCOMOMO ibérico que informa de la calidad del edificio al haber sido incluido en ese ilustre registro arquitectónico. Retrocediendo a las fechas previas al verano conviene recordar la charla que el arquitecto vallisoletano Óscar Miguel Ares nos dio para mostrar una interesante obra que ha sido galardonada habitualmente en la práctica totalidad de premios a los que ha optado. Unos meses después el COAA organizó un viaje para ver in situ muchas de esas obras mostradas por nuestro compañero, pudiendo corroborar la gran calidad de su trabajo. Tras el verano llegó un verdadero regalo para todos los que pudieron asistir a la charla de Carme Pinós, la arquitecta catalana que había sido socia y esposa del malogrado
Enric Miralles, quien ofreció una conferencia que de manera literal nos introdujo en su mundo, en sus postulados arquitectónicos, en sus problemas con la dislexia y que fue finalmente recompensada con un aplauso que pocas veces se ha visto en un acto de este tipo. Un auténtico lujo el haber podido contar con la presencia de Carme por nuestra tierra. Otro gran arquitecto nacido en Madrid pero que lleva en nuestra región desde hace más de 30 años, Enrique Perea, ofreció una interesante reflexión sobre la construcción en el medio rural y cómo intervenir en él no supone repetir pautas urbanas, si no que requiere un diálogo con el lugar que no tiene porqué caer en tópicos ni perder modernidad en las propuestas. Para acabar el año dos jóvenes arquitectas vinculadas a Asturias, las hermanas Andrea y Paula González, nos contaron sus experiencias en Suiza y Londres respectivamente, incidiendo en las diferencias principales que aprecian y cuáles son nuestros potenciales para ser competitivos en esos lugares atendiendo a la formación recibida en nuestro país.

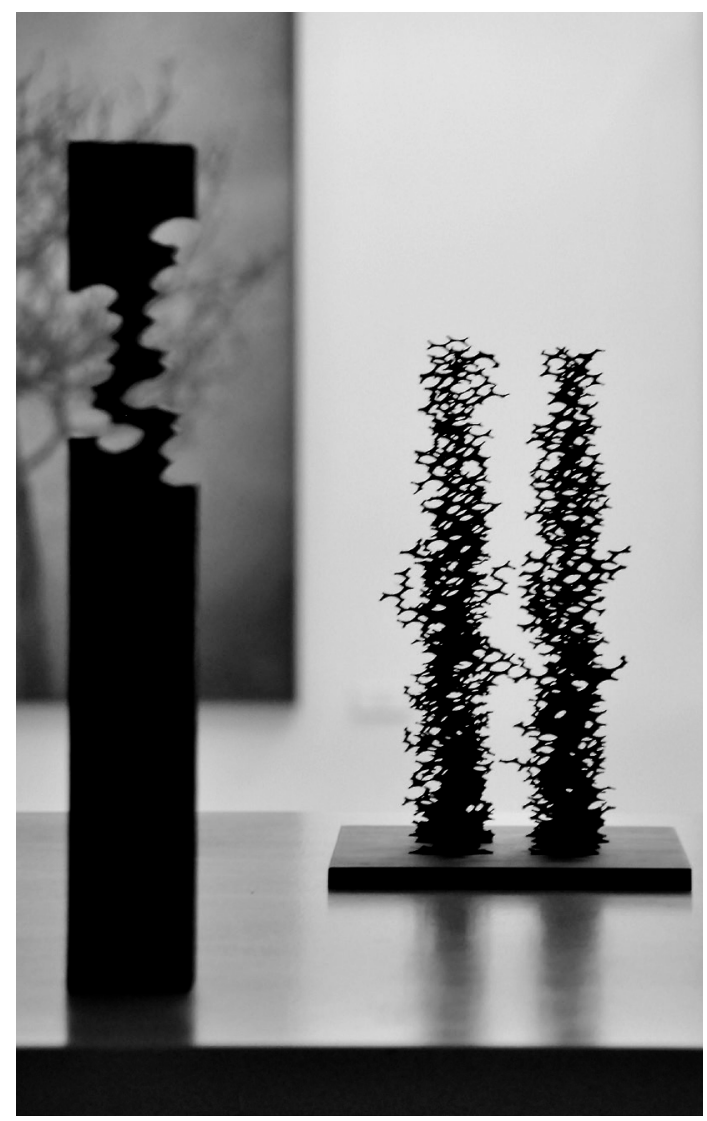

Fig. 1. Obras de Francisco Fresno en la exposición "Pasos, 19752019" realizada en la sede del COAA de Oviedo en marzo de 2019. Autor fotografía: Jose Ramón Puerto Álvarez. 


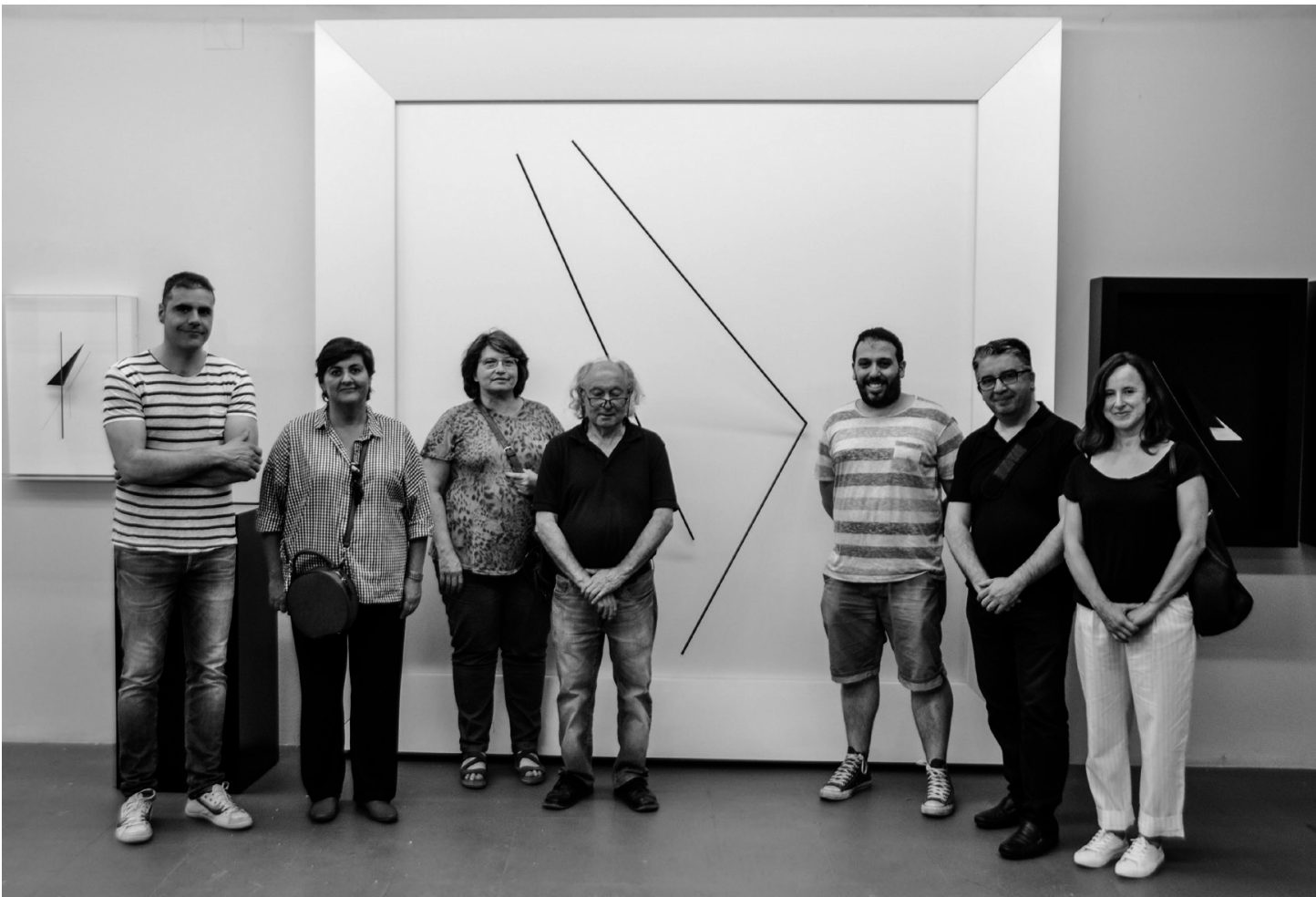

Fig. 2. Visita de colegiados del COAA al taller del escultor Herminio en La Caridad. Julio 2019. Fotografía: Jose Ramón Puerto Álvarez

En el periodo estival se aprovechó la menor actividad de esas fechas para organizar la visita al taller del escultor Herminio en La Caridad, quien nos regaló su tiempo y nos mostró con pasión su ingente obra, adornando con anécdotas cada nueva pieza que contemplábamos. Fue una jornada que terminó con la visita al centro cultural As Quintas que el escultor impulsó y donde se ha colocado una escultura suya en el exterior del conjunto.

No es posible realizar un repaso de nuestras actividades culturales del 2019 sin mencionar dos premios fundamentales en nuestro Colegio como son el Castelao, que se otorga para premiar a una trayectoria profesional y el $\mathrm{COAA}+10$, que galardona la obra que tras más de diez años de su realización sigue teniendo sus postulados arquitectónicos vigentes y su construcción ha permitido llegar hasta nuestros días de una forma adecuada. Fueron merecedores de los mismos respectivamente, el arquitecto Nicolás Arganza García (Tineo, 1932) y las Casas Cuartel de la Guardia Civil de Pola de Lena, Piedras Blancas y Luarca, realizadas todas ellas por los arquitectos Jose Manuel Caicoya (Gijón, 1951-2007) y Manuel García (La Habana, 1952).
El premio Castelao 2019 fue concedido a Nicolás Arganza por la "excelencia de su larga carrera profesional y su compromiso vital con la profesión”, según señaló el fallo del jurado. Cuenta en su haber con algunas de las obras más destacadas de la reciente historia de la arquitectura asturiana como son el edificio del Banco de España en Oviedo y los Colegios Meres y de las Teresianas, situados ambos en la periferia de la capital. Su época más fructífera, en la que se gestan sus proyectos de mayor calidad arquitectónica, fueron las décadas de 1970-1980. Fue presidente del Colegio Oficial de Arquitectos de Asturias y León durante los años 1975-76 y miembro de la Junta de Gobierno del COAA en 1982. $\mathrm{Su}$ trayectoria profesional abarca tanto lo público (como arquitecto asesor del Ayuntamiento de Bimenes) como lo privado, alternando la labor desde su despacho particular con el desempeño de su actividad para la empresa constructora Los Álamos, para la que realizó numerosos proyectos de viviendas de gran calidad. Con sus propuestas consiguió elevar el nivel de calidad de este tipo de edificaciones, tanto en lo constructivo como en lo arquitectónico. 


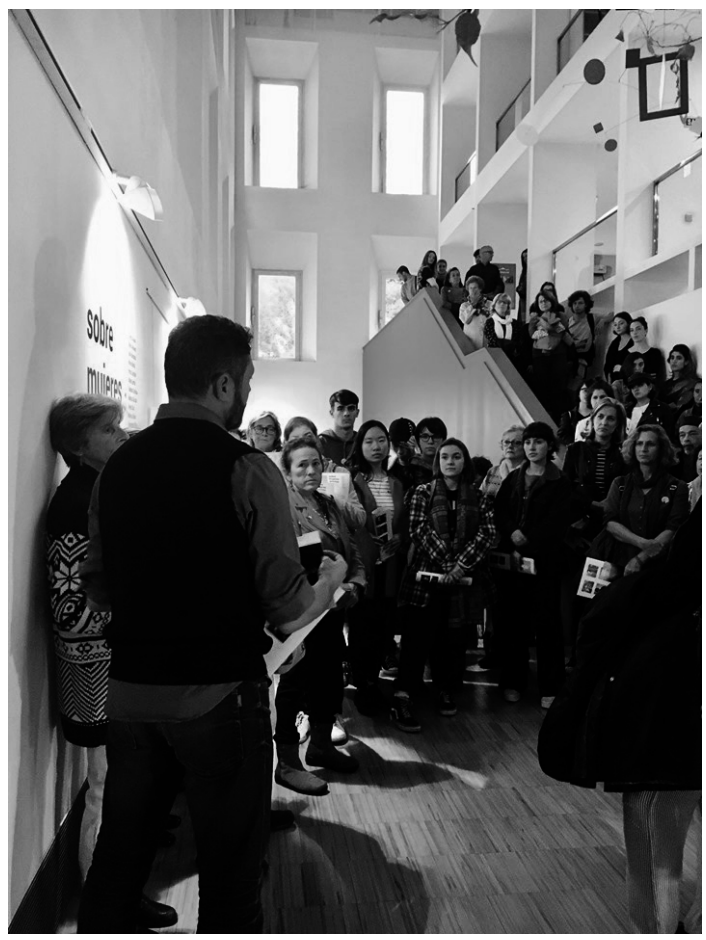

Fig. 3. Visita guiada a la exposición realizada en la sede del COAA de Oviedo "Sobre mujeres artistas" comisariada por Santiago Martínez. Octubre 2019. Autor fotografía: Jose Ramón Puerto Álvarez.

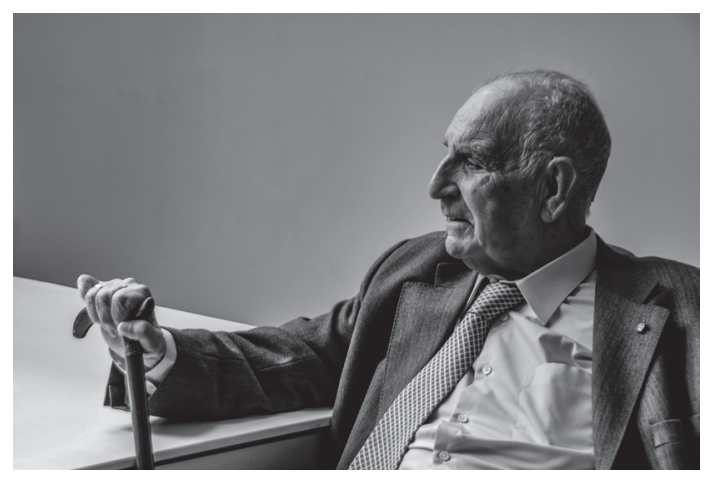

Fig. 4. El arquitecto Nicolás Arganza García, Premio Castelao 2019. Fotografía realizada en la sede del COAA de Oviedo en octubre de 2019 por Jose Ramón Puerto Álvarez

La entrega del premio se realizó el 22 de noviembre en uno de los edificios proyectados por Arganza, el Banco de España, contando con la asistencia del presidente del Principado de Asturias, Adrián Barbón, quien entregó el galardón al premiado junto con Alfonso Torre Solomando, decano del Colegio Oficial de Arquitectos de Asturias. Fue un acto emotivo en el que se premió al arquitecto, pero también al hombre, recordando su etapa de jugador profesional de rugby que le permitió defender la camiseta de la selección española. El cariño de la profesión y su extensa familia permitieron que el acto resultara entrañable.

El COAA+10, por su lado, valoró positivamente la propuesta de las tres casas cuartel citadas anteriormente por haber sido realizadas con "una singular impronta que ha sabido permanecer en el tiempo". El jurado del premio señaló que "supieron mantener su vigencia arquitectónica gracias a su claridad formal y volumétrica, así como una cierta atemporalidad en la resolución de sus fachadas". Éstas y otras características técnicas como la ausencia de aleros y la sencillez en la solución de antepechos, barandillas o el cierre de las parcelas, han hecho que las obras ofrezca "una imagen de contemporaneidad" distinta a la que se venía realizando en otros cuarteles. El estado de conservación de los edificios, señala el fallo, "puede calificarse como óptimo, aproximándose mucho al estado original" pese a algunos pequeños cambios, otro de los requisitos para obtener el reconocimiento.

Por la singularidad de los edificios premiados no se pudo realizar el acto de entrega en ninguno de ellos y fue la sede colegial de Oviedo la que lo acogió. Al mismo asistió una nutrida representación del cuerpo armado encabezados por el coronel jefe de la zona XIV de la Guardia Civil, Francisco Javier Almiñana, quien agradeció el reconocimiento y prometió seguir velando por el patrimonio premiado. El Decano del COAA señaló que "el premio parece cerrar un círculo, ya que la primera edición del premio Asturias de Arquitectura, convocado en 1983, fue para la casa cuartel de Piedras Blancas. Entonces, ya se valoró un edificio rompedor para su época, lejos de las tipologías al uso de las casas cuartel, y también muy innovador desde el punto de vista urbanístico". Manuel García, tras recibir el premio, recordó el buen hacer de su colega fallecido y también el trabajo imprescindible de quien había sido el aparejador de las obras, José Luis Pérez Espinosa, así como de las empresas que propiciaron la construcción de los edificios. Destacó el trabajo de conservación de la Guardia Civil durante todos estos años, y el respeto con que emprendió todas las obras y reformas que se necesitaron para poner al día los inmuebles.

El año terminó como empezó el anterior, con una exposición colectiva en la sede de Oviedo titulada "Nuestra otra obra" en la que se pudieron ver los trabajos realizados por colegiados fuera de la disciplina arquitectónica. 

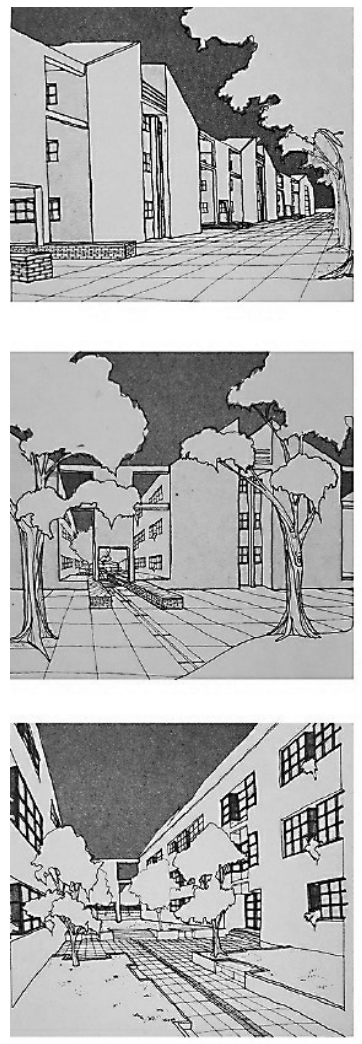

Perspectivas de la Casa Cuartel de Luarca incluidas en el proyecto

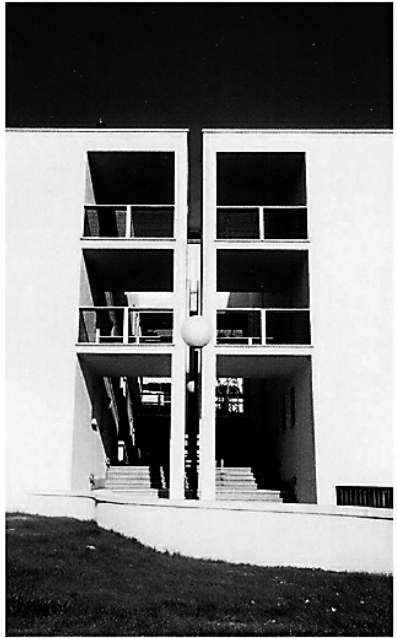

CASA CUARTEL PIEDRAS BLANCAS Edificio galardonado con el Premio Asturias de Arquitectura 1983. Está formado por la unión de dos bloques iguales de planta cuadrada con núcleo y patio interior, en torno al cual se disponen 4 viviendas. Al igual que los otros edificios, su imagen es deudora de una estudiada proporción y la correcta definición y colocación de huecos. Su claridad formal es atemporal, manteniendo por ello su vigencia arquitectónica, unida a un buen estado de conservación, sin patologías ni alteraciones de importancia.

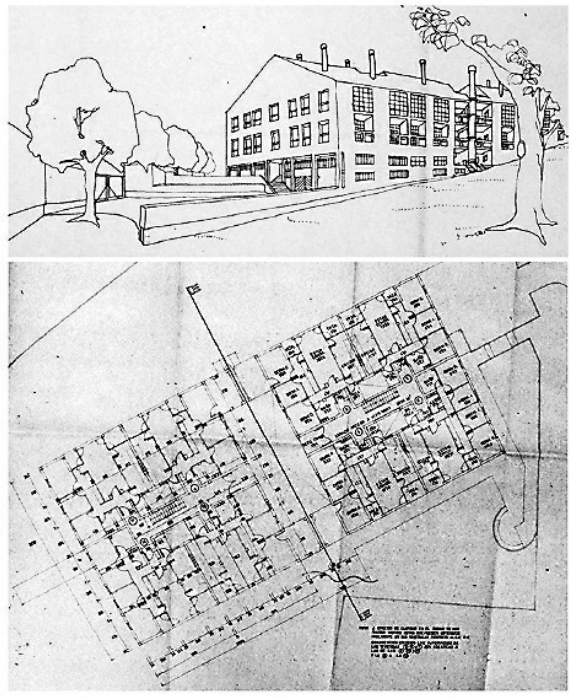

Perspectivas y planta de la Casa Cuartel de Piedras Blancas

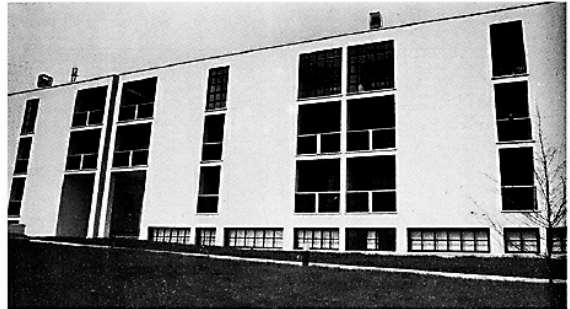

Fig. 5. Panel de la Casa Cuartel de la Guardia Civil en Piedras Blancas, Castrillón (1982), una de las tres galardonadas ex aequo con el premio COAA+10. Panel realizado por Valentín Arrieta Berdasco.

Más de 20 obras con diversidad de técnicas mostraron durante un mes la creatividad que podemos ofrecer cuando la funcionalidad no es uno de los elementos a resolver.

Sirvan estas notas como recordatorio de un fructífero año en el que la Cultura presidió muchos de nuestros actos lo que permitió que estos llegaran más a la sociedad, sumando sinergias que actúan de forma biunivoca y que nos retroalimentan para poder continuar y seguir trabajando ya en nuevas propuestas para el 2020. 